\title{
Asymptotic Performance Analysis of Successive Interference Cancellation with Dynamic User-Decoding Order
}

\author{
Francesc Molina, Student Member, IEEE, and Josep Sala-Álvarez, Senior Member, IEEE
}

\begin{abstract}
We address the evaluation of dynamic (estimationbased) user-decoding order in successive interference cancellation (SIC) in a proof-of-concept multiple access satellite setting in which many code-division multiple access users employ nonorthogonal spreading codes and the same encoder. We consider the practical setting where user symbol energies, unknown to the receiver, are estimated by preamble cross-correlations. Thereafter, SIC proceeds in non-increasing order of the former estimates. In contrast with small networks evaluated in the literature, we analyze the large-user regime and derive a simple system model to analyze the asymptotic network performance. Monte Carlo simulations validate our infinite-user analysis for hundreds of users and report the benefits of their application.
\end{abstract}

Index Terms-Non-orthogonal multiple access, successive interference cancellation, large-user analysis, user-decoding order.

\section{INTRODUCTION}

$\mathbf{I}$ $\mathrm{N}$ the latter years, several interference management techniques to mitigate multiple access interference (MAI) have been devised. Among them, SIC is a computationally affordable technique that operates sequentially over all users [1]. Likewise sequential algorithms, the order in which the system operates is a critical factor. With regard to SIC, however, setting the user-decoding order (UO) requires some knowledge of the received user strengths (powers or symbol energies) [2].

Theoretical analyses have revealed that SIC benefits from setting the UO in non-increasing order of received strengths. Thus, SIC always proceeds with the most powerful user at every stage [3], [4]. However, the practical application of the theoretical UO to machine-type communications is usually unachievable and needs some discussion. Firstly, energies of all users must be estimated and used to set the UO before SIC proceeds [5]. Secondly, the use of short packets may pose limitations: to setting the UO from short preamble lengths; and to network performance due to non-ideal encoding systems [6].

SIC has been simulated in many scenarios with successful results. In [7], SIC under different UO criteria is investigated. Closed-form expressions for the outage probability are derived for only two and three users by averaging results over all possible decode orderings [8], [9]. However, the previous analyses are infeasible when a large number of packets $K$ are ranked since the number of possible UOs grows as the

Work supported in part by Agencia Estatal de Investigación (AEI, Spain) and by European Regional Development Fund (ERDF) through project WINTER (TEC2016-76409-C2-1-R) and project RODIN (PID2019-105717RBC22), and in part by the Catalan administration (AGAUR) under Grant 2017 SGR 578 and predoctoral Fellowship 2019FI_B1 00085.

(Corresponding author: Francesc Molina.)

The authors are with the Department of Signal Theory and Communications, Universitat Politècnica de Catalunya, 08034 Barcelona, Spain (e-mail: francesc.molina@upc.edu; josep.sala@upc.edu). factorial $K$ !. Analytical findings for large-user satellite settings under perfect UO are reported in [10], [11]. Notwithstanding, a simple and accurate system model for dynamically ordered SIC handling a large number of users still remains to be found.

This letter concentrates on understanding the large-system structure of SIC under dynamic UO, rather than evaluating a small few-user network [7], [8], [9]. In lieu of the perfectly ordered SIC in [10], [11], we opted for an imperfectly ordered SIC that proceeds in non-increasing order of user-energy estimates. We consider non-ideal SIC operation for decoding and cancellation of users. In this respect, we adopt a general form to characterize both effects by taking their characteristics versus SINR after symbol despreading so that, our analysis remains valid regardless of the adopted encoding and cancellation systems. We tackle the yet unsolved performance analysis of dynamically ordered SIC through a deterministic model. We show that, when the number of users is large, we may adopt an average system model that weights the contributions of all users by the probabilities that each user is ordered at each position. In the large-user limit, their analytic closed forms are derived. Monte Carlo simulations have assessed our asymptotic system analysis for about a hundred of users.

For notation: The dot notation is used to indicate differentiation. Symbol denotes "statistically distributed as".

\section{SySTEM SET-UP}

We envisage a packet access satellite network where $K$ users transmit towards a common destination. Slotted time division is utilized to analyze the former scenario. At time slot $n, 1 \leq$ $k \leq K$ users transmit independent $n_{p}$-symbol packets $s_{k}[0 \leq$ $d<n_{p}$ ] made up of a known $n_{o}$-symbol preamble and a $n_{e}$-symbol payload, with $n_{p}=n_{o}+n_{e}$. Payload is generated by encoding information plus cyclic redundancy check (CRC) using the same channel encoder [4]. Users operate under power amplifiers with limited peak-to-average-power ratios. This may be achieved with code-division-multiple-access, and, to allow the simultaneous connection of many users, non-orthogonal spreading codes with the pseudonoise-like behavior described in [12] and period spanning many symbols are chosen. $c_{k, d}(t)$ denotes the spreading waveform of symbol $d$ for user $k$.

We center our analysis on reception of a satellite return link [4], [5], [10], wherein the aggregate received signal in a quasi-static channel with sufficiently long coherence time is, accounting for the $k$-th user received symbol energy $E_{\mathrm{r}}[k]$, the end-to-end delay $\tau[k]$, and $w(t)$ Gaussian noise:

$$
y(t)=\sum_{k=1}^{K} \sqrt{E_{\mathrm{r}}[k]} \sum_{d=0}^{n_{p}-1} s_{k}[d] c_{k, d}(t-\tau[k])+w(t) .
$$




\section{A. User-Energy Estimation}

The energies of all users are estimated at every time slot through a bank of single-user energy estimators that compute the cross-correlation of the known $n_{o}$-symbol preambles with the received (noisy) signal. Since users are superposed in the code domain, energy estimates are noisy and subject to MAI. In that respect, adopting long spreading codes with per-symbol processing gain $N$ entails that MAI be close to Gaussian at the despreader output [12], contributing thus as additional noise.

The accuracy of such estimates strongly depends on the SINR affecting each estimator, expressed respectively by each user's SINR after symbol despreading at the initial SIC stage:

$$
\Lambda[k] \triangleq \frac{E_{\mathrm{r}}[k]}{N_{t, n}^{0}[k]}=\frac{E_{\mathrm{r}}[k]}{N_{0}+\frac{\theta}{N} \sum_{i \neq k} E_{\mathrm{r}}[i]} .
$$

$N_{t, n}^{0}[k]$ is the noise plus MAI term on user $k$ with $N_{0}$ the noise power spectral density. Regarding MAI, $\frac{\theta}{N}$ is the average interuser per-symbol spreading code decorrelation factor, with $\frac{1}{N}$ that of slotted random binary spreading codes [11] $(\theta$ is also an ad-hoc decorrelation parameter used in unslotted systems with relevant $\tau[1 \leq k \leq K]$ dispersion [5]).

We assume that the channel of each user is stationary or varies sufficiently slow, so that, if users transmit the same energy during different time slots, the energy distribution $E_{\mathrm{r}}[1 \leq k \leq K]$ stays practically constant. In this case, energy estimates can be further improved by averaging estimates over $m$ time slots. At each time slot, we assume the user-energy estimations are affected by statistically independent noise plus MAI. Specifically, we consider the overall contribution of interfering users to user-preamble cross-correlations Gaussian and statistically independent between different time slots. Such is the case if, at each slot, users are received with different time variations (at least, in the order of chips), or if users randomize the carrier phase of each transmitted packet on a per-slot basis. Then, it is straightforward to prove, using [13], that the estimated symbol energy $\hat{E}_{\mathrm{r}}[k]$ follows a scaled noncentral chi-squared distribution with $2 m$ degrees of freedom and non-centrality parameter $2 m n_{o} \Lambda[k]$ :

$$
\hat{E}_{\mathrm{r}}[k] \sim N_{t, n}^{0}[k]\left(2 m n_{o}\right)^{-1} \cdot \mathcal{X}_{2 m}^{2}\left(2 m n_{o} \Lambda[k]\right) .
$$

\section{B. The User-Decoding Order}

For the sake of clarity, we define the user-index $1 \leq k \leq$ $K$, established in non-increasing order of the received user energies (4), and the user-order $1 \leq k^{\prime} \leq K$, set as a function of the estimated energies at time slot $n$ (5):

$$
\begin{aligned}
& E_{\mathrm{r}}[1] \geq \cdots \geq E_{\mathrm{r}}[k] \geq \cdots \geq E_{\mathrm{r}}[K], \\
& \hat{E}_{\mathrm{r}}[1] \geq \cdots \geq \hat{E}_{\mathrm{r}}\left[k^{\prime}\right] \geq \cdots \geq \hat{E}_{\mathrm{r}}[K] .
\end{aligned}
$$

Note that $1 \leq k \leq K$ (4) is fixed whereas $1 \leq k^{\prime} \leq K$ (5) depends on $n$ (dynamic). $k^{\prime}$ and $k$ can be related through the permutation

$$
\phi_{n}: k^{\prime} \in\{1, \ldots, K\} \longleftrightarrow k \in\{1, \ldots, K\},
$$

such that, $k=\phi_{n}\left[k^{\prime}\right]$ is used to obtain the index of the $k^{\prime}$-th ordered user, and vice-versa, $k^{\prime}=\phi_{n}^{-1}[k]$. Once the UO is set, SIC performance depends on the user-energy distribution $\tilde{E}_{\mathrm{r}}\left[1 \leq k^{\prime} \leq K\right]=E_{\mathrm{r}}\left[\phi_{n}\left[k^{\prime}\right]\right]$.

\section{SIC Operation}

After setting the UO, SIC performs $K$ stages starting from the user estimated with highest energy $k^{\prime}=1$. We adopt a SIC policy that, at the $k^{\prime}$-th stage, demodulates and decodes the $k^{\prime}$-th ordered user. After channel decoding, the CRC is used to determine packet success/error, after which, the user signal is regenerated and canceled only if successful decoding occurs. Then, for the obtained UO at time slot $n$, the SINR at the $k^{\prime}$-th SIC stage after symbol despreading is [11],

$$
\Gamma_{n}\left[k^{\prime}\right]=\frac{\tilde{E}_{\mathrm{r}}\left[k^{\prime}\right]}{N_{t, n}\left[k^{\prime}\right]}=\frac{\tilde{E}_{\mathrm{r}}\left[k^{\prime}\right]}{N_{0}+\xi_{\mathrm{prv}, n}\left[k^{\prime}\right]+\xi_{\mathrm{rem}, n}\left[k^{\prime}\right]}
$$

with the noise plus interference term at time slot $n N_{t, n}\left[k^{\prime}\right]$ written as the noise spectral density $N_{0}$ plus the sum of interference generated by users at stage $k^{\prime} . \xi_{\text {prv }, n}\left[k^{\prime}\right]$ denotes the aggregate interference of (previous) processed users $i<k^{\prime}$ and $\xi_{\mathrm{rem}, n}\left[k^{\prime}\right]$ that of remaining unprocessed users $i>k^{\prime}$.

To determine $\xi_{\text {prv }, n}\left[k^{\prime}\right]$ and $\xi_{\text {rem }, n}\left[k^{\prime}\right]$, we follow the $\Gamma$ based model in [5], [11] for decoding and cancellation. Therein, channel decoding is modeled with the known packet error rate (PER) vs. SNR curve PER $[\Gamma]$, while $0 \leq \varepsilon(\Gamma) \leq 1$ models the residual energy fraction after successful cancellation. Both effects are jointly analyzed in (8) by introducing one random variable $\epsilon_{i}(\Gamma)$ for the $\left(i<k^{\prime}\right)$-th user. That is, user $i$ with SINR $\Gamma=\Gamma_{n}[i]$ is either unsuccessfully decoded with probability PER $[\Gamma]$, for which, no cancellation occurs and $\epsilon_{i}(\Gamma)=1$; or successfully decoded with probability $\operatorname{PSR}[\Gamma] \triangleq$ $1-\operatorname{PER}[\Gamma]$ and imperfectly canceled with $\epsilon_{i}(\Gamma)=\varepsilon(\Gamma)$ (wherefore $\varepsilon(\Gamma) E_{\mathrm{r}}[i]$ uncanceled energy still remains). Then,

$$
\Gamma_{n}\left[k^{\prime}\right]=\frac{\tilde{E}_{\mathrm{r}}\left[k^{\prime}\right]}{N_{0}+\frac{\theta}{N} \sum_{i<k^{\prime}} \epsilon_{i}\left(\Gamma_{n}[i]\right) \tilde{E}_{\mathrm{r}}[i]+\frac{\theta}{N} \sum_{i>k^{\prime}} \tilde{E}_{\mathrm{r}}[i]} .
$$

Since the UO depends on energy estimates, the empirical evaluation of the average SIC performance needs exhaustive Monte Carlo simulations. Particularly, we are interested in two performance metrics. Firstly, the average number of packet errors user $k$ experiences over time computed using the longterm expression (9). Secondly, the average result over all users (10), namely the network PER:

$$
\begin{aligned}
\overline{\operatorname{per}}_{\mathrm{LT}}[k] & =\lim _{M \rightarrow \infty} M^{-1} \sum_{n=1}^{M} \operatorname{PER}\left[\Gamma_{n}\left[\phi_{n}^{-1}[k]\right],\right. \\
\overline{\operatorname{per}}_{\mathrm{LT}} & =K^{-1} \sum_{k=1}^{K} \overline{\operatorname{per}}_{\mathrm{LT}}[k] .
\end{aligned}
$$

\section{LARGE-USER ANALYSIS}

To gain further insight on SIC operation under dynamic UO, we assume the user-energy distribution $E_{\mathrm{r}}[1 \leq k \leq K]$ and the associated probability distribution of $\phi_{n}$ stay practically constant provided that the channel of each user is stationary. Then, equivalent expressions based on statistical rather than on the empirical averages (9) and (10) can be derived. Hereinafter, instead of considering the probability that a given UO (out of $K$ ! UOs) occurs, we consider that of user $k$ being ordered the $k^{\prime}$-th, computed detailedly in the Appendix and denoted from this point onwards user-order probability,

$$
p_{k, k^{\prime}} \triangleq \operatorname{Pr}\left[\phi_{n}^{-1}[k]=k^{\prime}\right]
$$


Moreover, when we focus on large networks for which the number of users $K$ and the spreading gain $N$ increase at a constant rate $\alpha \triangleq K / N$, some simplifications such as the SINR affecting the $k$-th user-energy estimator (2) can be performed:

$$
\Lambda[k]=\frac{E_{\mathrm{r}}[k]}{N_{0}+\alpha \theta \bar{E}-O\left(\frac{1}{N}\right)} \approx \frac{E_{\mathrm{r}}[k]}{N_{0}+\alpha \theta \bar{E}} .
$$

$\bar{E} \triangleq \frac{1}{K} \sum_{k=1}^{K} E_{\mathrm{r}}[k]$ is the average energy of all users, and $O\left(\frac{1}{N}\right)$ an infinitesimal term neglected at large $K$ (see the right part of (12)). Thus, since the denominator of (12) converges to a known value independently of $k$, the distribution of $\hat{E}_{\mathrm{r}}[k]$ in (3) can be simplified using $\beta \triangleq 2 m n_{o}\left(N_{0}+\alpha \theta \bar{E}\right)^{-1}$ as

$$
\hat{E}_{\mathrm{r}}[k] \sim \beta^{-1} \mathcal{X}_{2 m}^{2}\left(\beta E_{\mathrm{r}}[k]\right) .
$$

Note that $\hat{E}_{\mathrm{r}}[1 \leq k \leq K]$ follow independent non-identical noncentral chi-squared distributions with $2 m$ degrees of freedom. The validity of previous results is subject to having $\bar{E}$ finite (equivalently, finite $I_{\infty}$, according to [14, Ch. 2]).

\section{A. Large-User SIC Operation}

Herein, we determine an average system model for the system behavior described in Section II-C making use of the user-order probabilities first defined in (11).

The selection of such an average model follows from the large-system analysis of $N_{t, n}\left[k^{\prime}\right]$ (8). Firstly, as analyzed in Section II-C for a fixed distribution $\tilde{E}_{\mathrm{r}}\left[1 \leq k^{\prime} \leq K\right], N_{t, n}\left[k^{\prime}\right]$ is random due to non-ideal channel decoding. The law of large numbers, though, can be applied if $E_{\mathrm{r}}[1 \leq k \leq K]<\infty$ to show that $N_{t, n}\left[k^{\prime}\right]$ in (8) turns out to be deterministic as $K \rightarrow$ $+\infty$. Secondly, we have also empirically assessed, for several distributions $\tilde{E}_{\mathrm{r}}\left[1 \leq k^{\prime} \leq K\right]$, that the noise term that the user ordered at position $k^{\prime}$ experiences, $N_{t, n}\left[k^{\prime}\right]$, does not depend (as $K \rightarrow+\infty$ ) on the positions other users occupy. This means that $N_{t, n}\left[k^{\prime}\right]$ converges to an average utility as $K$ grows.

This has motivated the adoption of a bivariate analysis where the proposed system model only depends on both user $k$ and its position $k^{\prime}$, and not on the time slot index $n$. The validation of the adopted system model is addressed in Section V. Hence, we may consider, instead of the SINR in (7), an average SINR utility, denoted $\bar{\Gamma}\left[k, k^{\prime}\right]$, and computed as

$$
\bar{\Gamma}\left[k, k^{\prime}\right]=\frac{E_{\mathrm{r}}[k]}{\bar{N}_{t}\left[k, k^{\prime}\right]}=\frac{E_{\mathrm{r}}[k]}{N_{0}+\bar{\xi}_{\mathrm{prv}}\left[k, k^{\prime}\right]+\bar{\xi}_{\mathrm{rem}}\left[k, k^{\prime}\right]} .
$$

$\bar{\xi}_{\text {prv }}\left[k, k^{\prime}\right]$ and $\bar{\xi}_{\text {rem }}\left[k, k^{\prime}\right]$ are the corresponding previous and remaining interferences specified in (15) and (16).

1) Firstly, the average interference from processed users that user $k$ experiences when ordered the $k^{\prime}$-th is computed as

$$
\bar{\xi}_{\mathrm{prv}}\left[k, k^{\prime}\right]=\frac{\alpha \theta}{K} \sum_{i<k^{\prime}} \sum_{u \neq k} r(\bar{\Gamma}[u, i]) E_{\mathrm{r}}[u] p_{u, i},
$$

where $r(\Gamma) \triangleq 1-(1-\varepsilon(\Gamma)) \operatorname{PSR}[\Gamma]$ is the expectation of the random variable $\epsilon$. Specifically, we consider that $\bar{\xi}_{\text {prv }}\left[k, k^{\prime}\right]$ is given by the contribution of processing all users $u \neq k$ weighted by their user-order probabilities (11). That is, the interference contributed at every $1 \leq i<k^{\prime}$ SIC stage by user $u \neq k$ is the addition of: (i) $\operatorname{PER}[\bar{\Gamma}[u, i]] E_{\mathrm{r}}[u] p_{u, i}$ corresponding to a decoding failure of user $k$; plus (ii)
$\operatorname{PSR}[\bar{\Gamma}[u, i]] \varepsilon(\bar{\Gamma}[u, i]) E_{\mathrm{r}}[u] p_{u, i}$ for successful decoding. After a straightforward re-arrangement of terms, we get (15).

2) Secondly, the average remaining interference on user $k$ when ordered the $k^{\prime}$-th (third term of $\bar{N}_{t}\left[k, k^{\prime}\right]$ ) is given by the weighted sum of energies of users $u \neq k$. This is,

$$
\bar{\xi}_{\mathrm{rem}}\left[k, k^{\prime}\right]=\frac{\alpha \theta}{K} \sum_{i>k^{\prime}} \sum_{u \neq k} E_{\mathrm{r}}[u] p_{u, i} .
$$

Then, as argued at the beginning of Section III, equations (9)-(10) can be equivalently expressed by the expectations

$$
\overline{\operatorname{per}}[k]=\sum_{k^{\prime}=1}^{K} \operatorname{PER}\left[\bar{\Gamma}\left[k, k^{\prime}\right]\right] p_{k, k^{\prime}}, \overline{\operatorname{per}}=\frac{1}{K} \sum_{k=1}^{K} \overline{\operatorname{per}}[k] .
$$

\section{USER-ASYMPTOTIC ANALYSIS}

We analyze the asymptotic case in which both the number of users and the spreading gain go to infinity [3]. As usual, it is convenient to turn $k, k^{\prime}$ to the asymptotic variables

$$
t \triangleq \lim _{K \rightarrow+\infty} K^{-1} k \quad, \quad t^{\prime} \triangleq \lim _{K \rightarrow+\infty} K^{-1} k^{\prime}
$$

where, likewise, as in (4)-(5), $0 \leq t \leq 1$ is the user-index and $0 \leq t^{\prime} \leq 1$ the user-order. To proceed, we apply the transformations $\left\{k, k^{\prime}\right\} \rightarrow\left\{K t, K t^{\prime}\right\}$ on the previous variables to work with continuous functions (profiles) rather than with $k, k^{\prime}$ indexed variables. For instance, the received energy profile is

$$
E_{\mathrm{r}}(t) \triangleq \lim _{K \rightarrow+\infty} E_{\mathrm{r}}[K t] .
$$

The rest of profiles will be defined as they appear. Moreover, the probability user $k$ being ordered the $k^{\prime}$-th, now that of user $t=K^{-1} k$ being ordered $t^{\prime}=K^{-1} k^{\prime}$, is an infinitesimal quantity since the number of possible order positions increase with $K$ in the same order. As computed in the Appendix, $\mathrm{d} p\left(t, t^{\prime}\right)$ is asymptotically given, using $a\left(t, t^{\prime}\right)$ in (35), by the differential

$$
\mathrm{d} p\left(t, t^{\prime}\right)=a\left(t, t^{\prime}\right) \mathrm{d} t^{\prime}
$$

The asymptotic average SINR utility of user $t$ when ordered the $t^{\prime}$-th is expressed, in comparison with $\bar{\Gamma}\left[k, k^{\prime}\right]$ in (14), as

$$
\bar{\Gamma}\left(t, t^{\prime}\right)=\frac{E_{\mathrm{r}}(t)}{\bar{N}_{t}\left(t^{\prime}\right)}=\frac{E_{\mathrm{r}}(t)}{N_{0}+\bar{\xi}_{\mathrm{prv}}\left(t^{\prime}\right)+\bar{\xi}_{\mathrm{rem}}\left(t^{\prime}\right)} .
$$

Note therein that $\bar{N}_{t}\left[k, k^{\prime}\right]=\bar{N}_{t}\left[K t, K t^{\prime}\right] \rightarrow \bar{N}_{t}\left(t^{\prime}\right)$. That is, the corresponding noise plus interference term only depends on $t^{\prime}$ and not on $t$. This occurs because the contribution of the target user-energy $E_{\mathrm{r}}[K t]$ to (15)-(16) vanishes as $K$ increases. In this case, the MAI terms in the denominator of (21) are obtained by substituting the summations in (15)-(16) by integrals, with the differential $\frac{1}{K} \rightarrow \mathrm{d} u$, and $\mathrm{d} t^{\prime}$ in (20) as $\mathrm{d} \tau$. Thus, both terms become the double integrals

$$
\begin{aligned}
& \bar{\xi}_{\mathrm{prv}}\left(t^{\prime}\right)=\alpha \theta \int_{0}^{t^{\prime}} \int_{0}^{1} r(\bar{\Gamma}(u, \tau)) E_{\mathrm{r}}(u) a(u, \tau) \mathrm{d} u \mathrm{~d} \tau, \\
& \bar{\xi}_{\text {rem }}\left(t^{\prime}\right)=\alpha \theta \int_{t^{\prime}}^{1} \int_{0}^{1} E_{\mathrm{r}}(u) a(u, \tau) \mathrm{d} u \mathrm{~d} \tau .
\end{aligned}
$$

Finally, the user-asymptotic behavior of (17) results

$$
\overline{\operatorname{per}}(t)=\int_{0}^{1} \operatorname{PER}[\bar{\Gamma}(t, \tau)] a(t, \tau) \mathrm{d} \tau, \overline{\operatorname{per}}=\int_{0}^{1} \overline{\operatorname{per}}(t) \mathrm{d} t
$$




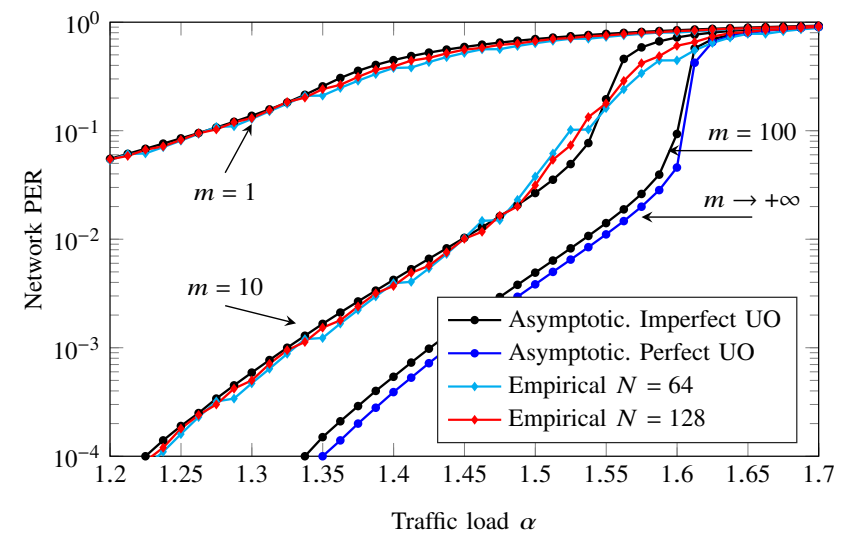

Fig. 1. Network PER versus traffic load $\alpha$. The asymptotic computations (circles) evaluate (24) and the empirical computations (diamond) (10). The asymptotic SIC behavior under perfect UO is taken from [11].

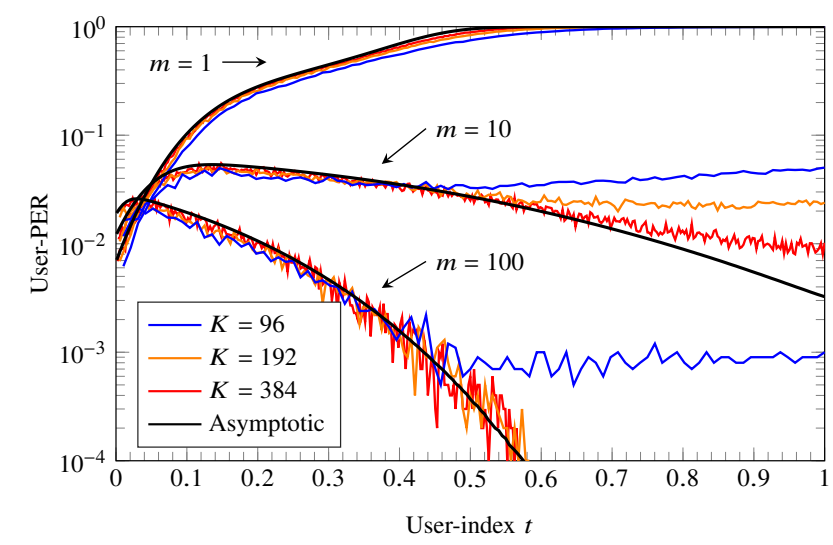

Fig. 2. User-PER versus user-index $t$ at $\alpha=1.50$. In black, the userasymptotic PER profile in the left part of (24). The long-term average PER in (9) is depicted for different numbers of users.

\section{Simulation Results}

This Section presents some numerical results. A modulation and coding scheme of rate $1 \mathrm{bps} / \mathrm{Hz}$ with preamble and payload lengths of $n_{o}=50$ and $n_{e}=450$ symbols is chosen. The PER vs. SNR characteristic is taken from [6] under rate $R=1$ and blocklength 450 . Results can be extended to other blocklengths, or to different modulations and error correcting codes as long as perfect knowledge of their PER vs. SNR curves is available. A simple $\Gamma$-independent model for the uncancelled energy $\varepsilon(\Gamma)=0.01$ is evaluated as in [4], [10]. The decorrelation factor $\theta$ is set to 1 [4]. Exponential userenergies are evaluated with $E_{\mathrm{r}}[k]=10 \exp \left(-1.5 \cdot K^{-1} k\right)$.

\section{A. Network and Individual Performances}

We validate the asymptotic system model in Section IV with the empirical model in Section II, by studying two magnitudes: the network PER and the user-PER.

We study in Fig. 1 the network PER versus the traffic load $\alpha$. As shown, severe performance degradation (black) w.r.t. perfect UO (dark blue) occurs for $m=1$. This is because, at the initial SIC stage, energy estimators operate at poor SINRs, especially so for the last users in $0 \leq t \leq 1$. It is shown that

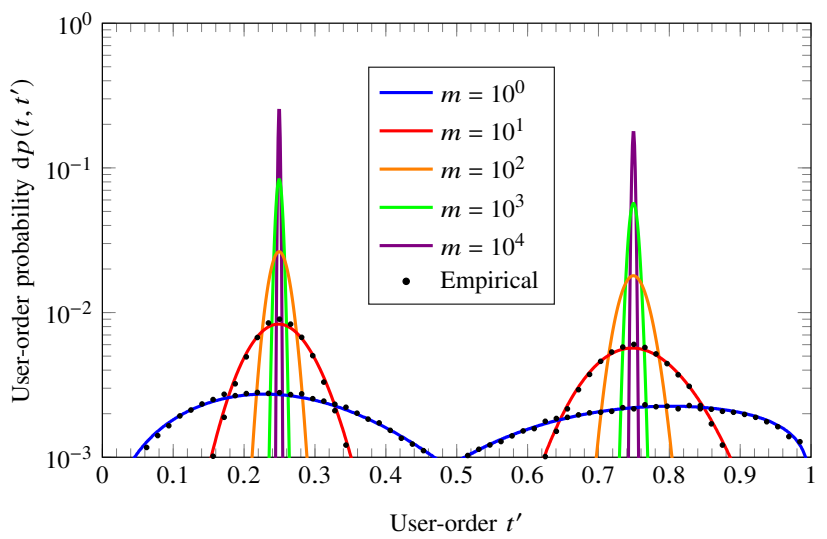

Fig. 3. Asymptotic order probabilities of users $t_{1}=0.25$ and $t_{2}=0.75$ when ordered at $t^{\prime}$, for several $m$ and $\alpha=1.50$. Empirical probabilities (black dots) are computed under $10^{5}$ averaged Monte Carlo runs and $K=64$ users.

for increasing $m$, the performance of SIC with perfect UO is approached. Empirical results under the system model in Section II evidence that the asymptotic PER in the right part of (24) is validated through (9) for about 100 users.

Secondly, we select the traffic load $\alpha=1.50$ and validate the asymptotic user-PER under different numbers of users. As shown in Fig. 2, the behavior of the empirical PER profile (markers) is accurately predicted by the asymptotic profile (black), especially for strong users (left-hand side of the $t$ interval $[0,1])$. It can also be observed that the degree of accuracy of the asymptotic user-PER prediction w.r.t. the empirical PER that weak users display (right-hand side of the $t$-interval $[0,1])$ increases as $K$ grows larger. This fact is well represented in Fig. 2 for the case $m=10$. Although the userPER needs more users to validate the asymptotic equations, this causes negligible degradation of the network PER.

\section{B. User-Order Probabilities}

In Fig. 3, we show the asymptotic probabilities, computed using $10^{3}$ equally-spaced samples of $t^{\prime}$ with $\mathrm{d} t^{\prime}=10^{-3}$, particularized for the user indices $t_{1}=0.25$ and $t_{2}=0.75$. As shown, for users $t_{1}, t_{2}$, their most probable UO values tend to $t_{1 \leq i \leq 2}$ almost surely as $m \rightarrow+\infty$. Specifically, when user-energies are estimated without taking into account previous estimations, that is, $m=1$ depicted in blue, $p\left(t_{1 \leq i \leq 2}, t^{\prime}\right)$ appear under a wide range $\left(r_{i} \triangleq\left|t_{1}^{\prime}-t_{2}^{\prime}\right|\right.$ such that $\left.p\left(t_{i}, t_{1}^{\prime}\right)=p\left(t_{i}, t_{2}^{\prime}\right)=p\right)$. At the cutoff probability $p=10^{-3},\left\{r_{1}, r_{2}\right\}=\{0.42,0.48\}$. This is because the last users are estimated under worse SINRs, and their estimations are improved as more estimations are averaged, viz: $\left\{r_{1}, r_{2}\right\}=\{0.20,0.26\}$ at $m=10$ (red), and $\left\{r_{1}, r_{2}\right\}=\{0.08,0.11\}$ at $m=100$ (orange). Moreover, the empirical probabilities scaled by $K \mathrm{~d} t^{\prime}$ (black circles) validate our asymptotic computation for only 64 users.

An additional simulation, not included for lack of space, validates that the asymptotic $K$-independent kernel $a\left(t, t^{\prime}\right)$ in (35) contains around the bisection $t^{\prime}=t$ the most probable UO position $\left(t^{\prime}\right)$ user $t$ occupies. The derivation of $a\left(t, t^{\prime}\right)$ is of relevant interest because it allows the fast computation of empirical probabilities regardless of the network size $K$. Thus, for a $K$-user network, $p_{k, k^{\prime}} \approx a\left(K^{-1} k, K^{-1} k^{\prime}\right) \cdot K^{-1}$. 


\section{CONCLUSION}

We have analyzed the dynamic decoding order of a SIC receiver in terms of its average PER and individual userPER performances in a large-user non-orthogonal multiple access scenario where users share the same encoding system. A practical SIC that sets the decoding order according to instantaneous energy estimations has been adopted. We show that the impact of user-decoding order on network performance can be assessed by deriving a system model that averages PER magnitudes according to the probabilities that each user be ordered at any position. Analytic expressions for these probabilities are obtained in the infinite-user case, and have revealed a decay with respect to the number of users $K$ with order $O\left(K^{-1}\right)$. Moreover, large- and asymptotic-system expressions are derived and validated using exhaustive Monte Carlo simulations for hundreds of users. A very accurate approximation to real SIC's performance at large $K$ is obtained from their asymptotic behavior.

\section{APPENDIX: COMPUTATION OF USER-ORdER PROBABILITy}

We seek an explicit equation for the probability user $k$ is ordered the $k^{\prime}$-th

$$
p_{k, k^{\prime}}=\operatorname{Pr}\left[\phi_{n}^{-1}[k]=k^{\prime}\right] .
$$

Since energy estimations are mutually independent, we focus on the computation of the user-order probabilities for a single user and extend the result to all users later on.

We first compute the distribution of $\phi_{n}^{-1}[k]$ when $\hat{E}_{\mathrm{r}}[k]=x$ is given, and denote it by $\phi_{n}^{-1}[k] \mid\left\{\hat{E}_{\mathrm{r}}[k]=x\right\}$. In this case, the index of user $k$ in the UO can be computed as a function of the number of users estimated with energies higher than that of user $k$. Mathematically,

$$
\phi_{n}^{-1}[k] \mid\left\{\hat{E}_{\mathrm{r}}[k]=x\right\} \triangleq 1+\sum_{i \neq k} \mathcal{B}_{i},
$$

with $\mathcal{B}_{i} \forall i \neq k$ independent Bernoulli random variables with success probabilities $q_{i} \triangleq \operatorname{Pr}\left[\hat{E}_{\mathrm{r}}[i]>x\right]$, actually, equal to 1 if user $i$ is estimated with higher energy than user $k . q_{i}$ is the tail distribution of a noncentral chi-squared distribution expressed using the Marcum Q-function $Q_{m}(a, b)$ as

$$
q_{i} \mid\left\{\hat{E}_{\mathrm{r}}[k]=x\right\} \triangleq Q_{m}\left(\sqrt{\beta E_{\mathrm{r}}[i]}, \sqrt{\beta x}\right) .
$$

In this case, since (26) is the sum of independent nonidentically-distributed Bernoulli random variables, it follows a Poisson Binomial distribution. If $K$ is large enough, we can invoke the Lyapunov central limit theorem to conclude that $\phi_{n}^{-1}[k] \mid\left\{\hat{E}_{\mathrm{r}}[k]=x\right\} \sim \mathcal{N}\left(\mu_{k}, \sigma_{k}^{2}\right)$, with

$$
\mu_{k} \triangleq 1+\sum_{i \neq k} q_{i}, \quad \sigma_{k}^{2} \triangleq \sum_{i \neq k} q_{i}\left(1-q_{i}\right) .
$$

Therefore, $\operatorname{Pr}\left[\phi_{n}^{-1}[k] \mid\left\{\hat{E}_{\mathrm{r}}[k]=x\right\}=k^{\prime}\right] \approx f_{\mathcal{N}}\left(k^{\prime}\right)$, with $f_{\mathcal{N}}(x)$ the Gaussian density of mean $\mu_{k}$ and variance $\sigma_{k}^{2}$.

Finally, we recover the initial case where $\hat{E}_{\mathrm{r}}[k]$ is random. Then, $p_{k, k^{\prime}}$ in (25) is computed by the expectation

$$
p_{k, k^{\prime}}=\int_{0}^{\infty} f_{\mathcal{N}}\left(k^{\prime} ; \mu_{k}(x), \sigma_{k}^{2}(x)\right) \cdot f_{\hat{E}_{\mathrm{r}}[k]}(x) \mathrm{d} x .
$$

$f_{\hat{E}_{\mathrm{r}}[k]}(x), x \geq 0$ is the density of (13), and $\bar{F}_{\hat{E}_{\mathrm{r}}[k]}(x)$ is the associated tail distribution used in the following subsection.

\section{A. Asymptotic User-Order Probability}

In the infinite-user case, we may define

$$
\phi_{n}^{-1}(t)=\lim _{K \rightarrow+\infty} K^{-1} \phi_{n}^{-1}[K t] .
$$

$\phi_{n}^{-1}(t) \mid\left\{\hat{E}_{\mathrm{r}}(t)=x\right\}, 0 \leq t \leq 1$ are identically distributed Gaussian distributions with the same mean $\bar{\mu} \triangleq \lim _{K \rightarrow \infty} K^{-1} \mu_{K t}$ and variance $\bar{\sigma}^{2} \triangleq \lim _{K \rightarrow \infty} K^{-2} \sigma_{K t}^{2}=0$. Then,

$$
\phi_{n}^{-1}(t) \mid\left\{\hat{E}_{\mathrm{r}}(t)=x\right\} \sim \delta(t-\bar{\mu}(x)),
$$

with $\delta(x)$ the Dirac delta function, and

$$
\bar{\mu}(x)=\int_{0}^{1} Q_{m}\left(\sqrt{\beta E_{\mathrm{r}}(\tau)}, \sqrt{\beta x}\right) \mathrm{d} \tau .
$$

In the user-asymptotic case, we may compute the probability that user $t$ is ordered the $t^{\prime}$-th turned to the differential

$$
\begin{aligned}
\mathrm{d} p\left(t, t^{\prime}\right) & \triangleq \operatorname{Pr}\left[t^{\prime}-\mathrm{d} t^{\prime} / 2 \leq \phi_{n}^{-1}(t) \leq t^{\prime}+\mathrm{d} t^{\prime} / 2\right] \\
& =\mathrm{d} t^{\prime} \int_{0}^{\infty} \delta\left(t^{\prime}-\bar{\mu}(x)\right) f_{\hat{E}_{\mathrm{r}}(t)}(x) \mathrm{d} x,
\end{aligned}
$$

which can be simplified after some manipulations, and then, (34) obeys $\mathrm{d} p\left(t, t^{\prime}\right)=a\left(t, t^{\prime}\right) \mathrm{d} t^{\prime}$, with

$$
a\left(t, t^{\prime}\right) \triangleq \nabla_{t^{\prime}} \bar{F}_{\hat{\mathrm{E}}_{\mathrm{r}}(t)}\left(\bar{\mu}^{-1}\left(t^{\prime}\right)\right)=-\left.\frac{f_{\hat{E}_{\mathrm{r}}(t)}(x)}{\dot{\bar{\mu}}(x)}\right|_{x=\bar{\mu}^{-1}\left(t^{\prime}\right)} .
$$

$\bar{\mu}^{-1}\left(t^{\prime}\right)$ is the $x_{*}$ solution of $\bar{\mu}\left(x_{*}\right)=t^{\prime}$. Note that (35) remains constant with $K$ whereas $\mathrm{d} p\left(t, t^{\prime}\right)$ vanishes as $K^{-1} \rightarrow \mathrm{d} t^{\prime}$.

\section{REFERENCES}

[1] J. G. Andrews, "Interference cancellation for cellular systems: a contemporary overview," IEEE Wireless Commun., vol. 12, no. 2, pp. 19-29, Apr. 2005.

[2] Y. Zhang, K. Peng, Z. Chen, and J. Song, "SIC vs. JD: Uplink NOMA techniques for M2M random access," in 2017 IEEE Int. Conference on Communications (ICC), May 2017, pp. 1-6.

[3] D. V. Djonin and V. K. Bhargava, "Asymptotic analysis of the conventional decision feedback receiver in fading channels," IEEE Trans. Wireless Commun., vol. 2, no. 5, pp. 1066-1078, Sep. 2003.

[4] J. Sala-Álvarez, F. Rey, J. Villares, and F. Molina, "Minimum PER user-energy profile for massive SIC receivers under an average energy constraint," in 2017 IEEE 18th Int. Workshop on Signal Processing Advances in Wireless Communications (SPAWC), Jul. 2017, pp. 1-6.

[5] R. De Gaudenzi, O. Del Río Herrero, and G. Gallinaro, "Enhanced spread ALOHA physical layer design and performance," Int. Journal on Satellite Communications and Networking, vol. 32, no. 6, pp. 457-473, Nov 2014.

[6] Y. Polyanskiy, H. V. Poor, and S. Verdú, "Channel coding rate in the finite blocklength regime," IEEE Trans. Inf. Theory, vol. 56, no. 5, pp. 2307-2359, May 2010.

[7] M. Mohammad and R. M. Buehrer, "The effects of ordering criteria in linear successive interference cancellation in CDMA systems," IEEE Trans. Wireless Commun., vol. 7, no. 11, pp. 4128-4132, Nov. 2008.

[8] Y. Gao, B. Xia, Y. Liu, Y. Yao, K. Xiao, and G. Lu, "Analysis of the dynamic ordered decoding for uplink NOMA systems with imperfect CSI," IEEE Trans. Veh. Technol., vol. 67, no. 7, pp. 6647-6651, Jul. 2018.

[9] Y.Gao, B. Xia, K. Xiao, Z. Chen, X. Li, and S.Zhang, "Theoretical analysis of the dynamic decode ordering SIC receiver for uplink NOMA systems," IEEE Commun. Lett., vol. 21, no. 10, pp. 2246-2249, Oct. 2017.

[10] F. Collard and R. De Gaudenzi, "On the optimum packet power distribution for spread ALOHA packet detectors with iterative successive interference cancelation," IEEE Trans. Wireless Commun., vol. 13 , no. 12, pp. 6783-6794, Dec. 2014.

[11] J. Sala, J. Villares, and F. Rey, "Asymptotic and finite user PER analysis of successive interference cancellation for DS-CDMA," IEEE Commun Lett., vol. 15, no. 11, pp. 1145-1147, Nov. 2011.

[12] S. Verdú, Multiuser detection. Cambridge University Press, 1998.

[13] S. Kay, Fundamentals of statistical signal processing: Estimation theory. Prentice Hall International Editions, Englewood Cliffs, NF, 1993.

[14] M. Haenggi and R. K. Ganti, "Interference in large wireless networks," Foundations and Trends in Networking, vol. 3, no. 2, pp. 127-248, 2009. 